\title{
In situ thin-film texture determination
}

\author{
Dmitri Litvinov, ${ }^{\text {a) }}$ Thomas O'Donnell, and Roy Clarke \\ Randall Laboratory of Physics, University of Michigan, Ann Arbor, Michigan 48109-1120
}

(Received 22 June 1998; accepted for publication 12 November 1998)

\begin{abstract}
A kinematic theory of reflection high energy electron diffraction (RHEED) is presented for textured polycrystalline thin films. RHEED patterns are calculated for arbitrary texture situations and for any general crystallographic orientation that may be encountered in thin-film growth. It is shown that the RHEED pattern can be used as a fast and convenient tool for in situ texture characterization. The approach also permits quantitative extraction of angular dispersion parameters which are useful for optimizing thin-film growth conditions. (C) 1999 American Institute of Physics.
\end{abstract}

[S0021-8979(99)04904-X]

\section{INTRODUCTION}

Reflection high energy electron diffraction (RHEED) has long been a powerful tool for thin-film surface analysis. However, it is mostly used for characterization of single crystal epitaxial films. ${ }^{1-4}$ While there are many other thinfilm microstructures of practical interest, such as textured polycrystalline films, ${ }^{5-7}$ these cases are only recently being addressed. The practical value of RHEED techniques to texture determination in polycrystalline thin film has recently been emphasized by Andrieu et al. ${ }^{8}$ These authors calculated RHEED patterns corresponding to specific textures and symmetries (bcc) and showed that it is possible to extract quantitative information on the angular distribution of grains within the film. However, in order to make this approach broadly useful to a wide range of realistic thin-film growth situations, further analysis is necessary that would provide a general formalism covering all possible textures and, in general, any preferred crystallite alignment (e.g., mosaic crystals).

In this article we develop an analytical treatment of RHEED patterns generated by textured thin films. While the theoretical expressions presented here are useful for analyzing the quantitative details of the pattern, including the angular dispersion of crystallites, the approach is most useful for providing a fast and convienient characterization of the texture, including the direction of the texture axis and the symmetry of the preferred orientation.

\section{THEORY}

This work presents a kinematic theory of RHEED for textured polycrystalline materials. We show that the presence of texture gives rise to distinct RHEED characteristics, and that these data can be used to extract useful information about crystallographic orientation of the texture axis and its angular dispersion. To preserve generality, the texture is assumed to be not ideal but rather to have some angular distribution. As an approximation, electron beams diffracted from different crystallites are assumed to have random phases so that the intensities of the beams diffracted from different

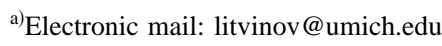

crystallites add. This is justified by the short transverse coherence length in a typical RHEED configuration $(\sim 100 \AA)$ compared to typical crystallite sizes (>500 $⿱$ A). Furthermore, due to the surface roughness of real polycrystalline films, the case of 3D transmission diffraction is assumed.

In the Ewald construction the diffraction condition is given by:

$$
\mathbf{K}_{\mathrm{e}}^{\prime}-\mathbf{K}_{\mathrm{e}}=\mathbf{G}
$$

where $\mathbf{K}_{\mathbf{e}}^{\prime}$ and $\mathbf{K}_{\mathbf{e}}$ are the scattered and the incident wave vectors of the electron, respectively, and $\mathbf{G}$ is a reciprocal lattice vector

$$
\mathbf{G}=h \mathbf{a}^{*}+k \mathbf{b}^{*}+l \mathbf{c}^{*} .
$$

For typical electron energies used in RHEED $(\sim 10-30 \mathrm{keV})$ the wavelength of the electrons, $\lambda_{e}(\sim 0.1-0.2 \AA)$ is $\ll a$ (where $a$ is the crystal lattice parameter). It follows that for low orders of diffraction, or $\left(a / \lambda_{e}\right)^{2} \gg h^{2}+k^{2}+l^{2}$, the electron wave vector $K_{e} \gg G$. Under these conditions the Ewald surface can be approximated by a plane and the diffraction condition then becomes

$$
\mathbf{K}_{\mathrm{e}} \mathbf{G}=\mathbf{0} .
$$

Equation (2.3) states that all reciprocal lattice vectors satisfying the diffraction condition lie in a plane perpendicular to $\mathbf{K}_{\mathbf{e}}$. For our purposes only the plane intersecting the termination of the vectors $\mathbf{K}_{\mathbf{e}}$ and $\mathbf{K}_{\mathbf{e}}^{\prime}$ will be considered, corresponding to the Ewald construction shown in Fig. 1.

\section{A. Reciprocal lattice of a polycrystalline film with a texture}

It is useful to develop a picture of the reciprocal lattice of a polycrystalline film. In the case of polycrystalline films without any texture or preferred crystallite alignment, the reciprocal "lattice" can be thought of as a set of concentric spheres (with radii equal to $G=\sqrt{h^{2}+k^{2}+l^{2}}$ in the case of orthogonal lattices). For ideal texture, i.e., when there is no dispersion in the texture orientation, the reciprocal lattice consists of a set of points along the texture direction superposed with a set of concentric rings lying in discrete parallel planes perpendicular to the texture direction [see Fig. 2(a)]. In the case where there is angular dispersion in the texture 


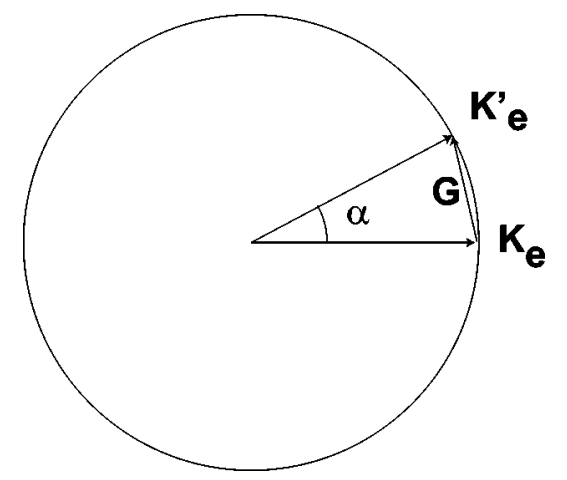

FIG. 1. Ewald construction for the diffraction condition.

orientation, the reciprocal lattice can be described by a set of concentric domes along the texture direction superposed with a set of concentric circular bands; both domes and circular bands being parts of concentric spheres with common center at the origin of the reciprocal lattice [see Fig. 2(b)].

As mentioned above, the RHEED pattern is defined by the intersection of the Ewald sphere (approximated by a plane in this case) and the reciprocal lattice. Symmetry dictates that if the RHEED pattern does not change as the sample is rotated around an axis perpendicular to the film surface, the texture axis must be oriented perpendicular to the film. It follows that, in this case, the intersection of an Ewald surface with domes along the texture direction will appear as an arc symmetric with respect to the $z$ axis while all other diffraction features will appear as partial rings symmetrical around the $z$ axis with minima along the $z$ axis. This suggests a simple way of determining the texture of the film: if the RHEED pattern is invariant with respect to the sample

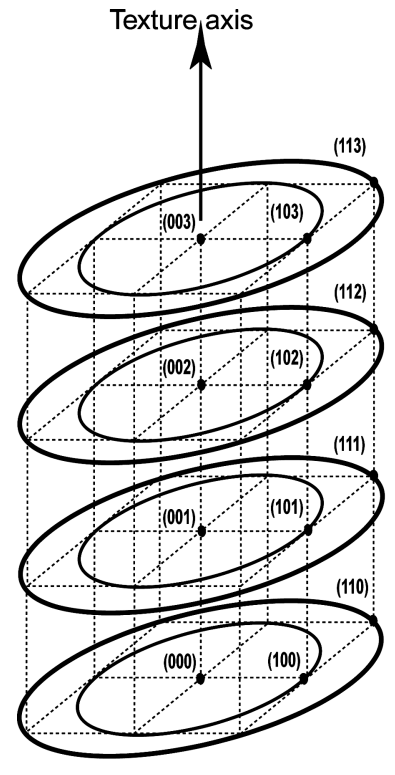

(a)

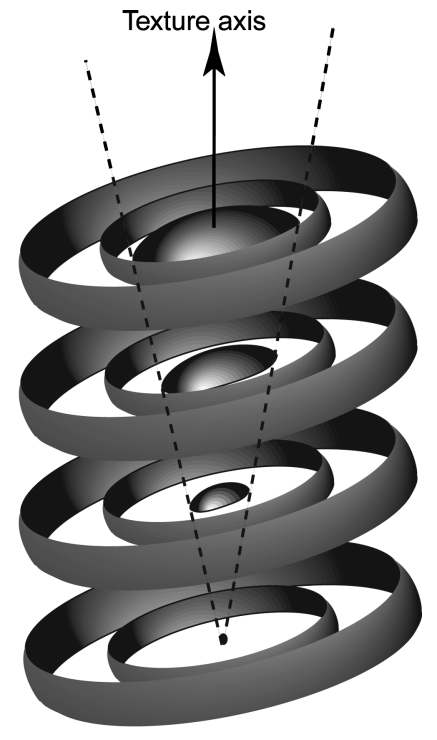

(b)
FIG. 2. (a) Reciprocal lattice of a polycrystalline film with an ideal texture. (001) texture in a film with a simple cubic lattice is illustrated. The rings represent the trajectories of reciprocal lattice points upon rotation around the texture axis; (b) sketch of the reciprocal lattice of a polycrystalline film with a non-ideal texture.

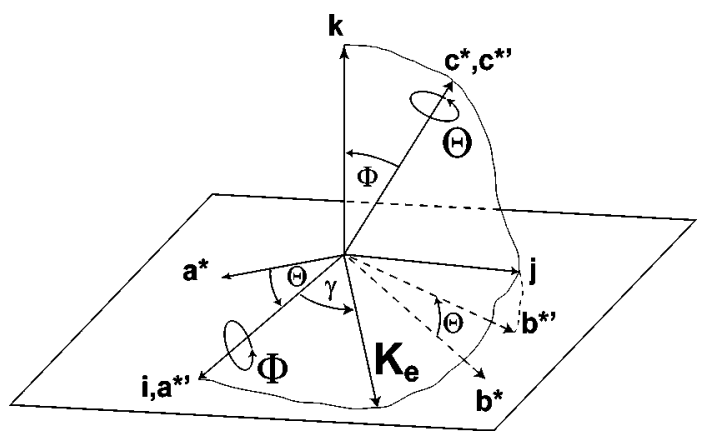

FIG. 3. Thin-film coordinate axis geometry. The thin curved lines indicate which vectors are coplanar. For example, $\mathbf{c}^{*^{\prime}}$ and $\mathbf{b}^{*^{\prime}}$ lie in the $k j$ plane.

rotation around an axis perpendicular to the film surface and an arc centered around the $z$ axis is present, the rotation axis corresponds to a texture direction in the film.

In general, if the texture axis is at some nonzero angle with respect to the film surface normal, the RHEED pattern will change as the sample is rotated around the axis perpendicular to the film surface. However, the RHEED pattern should be symmetric when the texture axis is parallel to the plane of specular diffraction (a plane spawned by $\mathbf{K}_{\mathbf{e}}$ and the normal to the film surface). It will be shown below that these qualitative arguments are supported by detailed analytical expressions for RHEED patterns in arbitrary geometries.

\section{B. Case of orthogonal crystal axes}

\section{1. (001) texture}

Without loosing generality, let us first consider a case where the texture is oriented along the (001) crystal direction. We recall that such a film is a collection of crystallites with one of the crystallographic directions fixed which defines the texture and these crystallites are free to rotate around the texture direction. It will be shown subsequently how this case can be extended to an arbitrary texture direction. We shall choose a laboratory frame $(\mathbf{i}, \mathbf{j}, \mathbf{k})$ such that $\mathbf{k}$ is normal to the substrate surface and the texture direction lies in the ( $j k)$ plane (see Fig. 3). We label by $\Phi$ the angle between $\mathbf{k}$ and texture orientation. $\Theta$ is the angle of rotation around texture direction and it is the only parameter in the problem that can be adjusted so that the diffraction condition (2.3) is satisfied (we assume a uniform and continuous distribution of $\Theta$ such that one can always find a crystallite with a given value of $\Theta$ ).

First, to solve the diffraction equation (2.3), we need to determine the coordinates of an arbitrary reciprocal vector $\mathbf{G}(h k l)$ in the laboratory frame such that

$$
\mathbf{G}=G_{x} \mathbf{i}+G_{y} \mathbf{j}+G_{z} \mathbf{k} \text {. }
$$

The coordinate transformation to express $\left(G_{x}, G_{y}, G_{z}\right)$ in terms of $(h k l)$ is obtained by performing two rotations of the reciprocal lattice frame: the first is a counterclockwise rotation around $\mathbf{c}^{*}$ by angle $\Theta$ that makes $\mathbf{a}^{*}$ and $\mathbf{i}$ coincide, and the second is a counterclockwise rotation around $\mathbf{i}$ by angle $\Phi$ : 


$$
\begin{aligned}
& R_{\mathbf{c}^{*}, \Theta}=\left(\begin{array}{ccc}
\cos \Theta & -\sin \Theta & 0 \\
\sin \Theta & \cos \Theta & 0 \\
0 & 0 & 1
\end{array}\right), \\
& R_{\mathbf{i}, \Phi}=\left(\begin{array}{ccc}
1 & 0 & 0 \\
0 & \cos \Phi & -\sin \Phi \\
0 & \sin \Phi & \cos \Phi
\end{array}\right) .
\end{aligned}
$$

The resulting transformation is the product of the two rotations in the specific order given:

$$
J_{h k l \rightarrow G_{x} G_{y} G_{z}}=R_{\mathbf{i}, \Phi} R_{\mathbf{c}^{*}, \Theta} .
$$

Then the $\left(G_{x}, G_{y}, G_{z}\right)$ coordinates are given by

$$
\begin{aligned}
\left(\begin{array}{c}
G_{x} \\
G_{y} \\
G_{z}
\end{array}\right)= & \frac{2 \pi}{a}\left(\begin{array}{ccc}
\cos \Theta & -\sin \Theta & 0 \\
\cos \Phi \sin \Theta & \cos \Phi \cos \Theta & -\sin \Phi \\
\sin \Phi \sin \Theta & \sin \Phi \cos \Theta & \cos \Phi
\end{array}\right) \\
& \times\left(\begin{array}{c}
h \\
k \\
l
\end{array}\right)
\end{aligned}
$$

Consider now the case of a near grazing incidence electron beam so that $\mathbf{K}_{\mathbf{e}}$ can be expressed as

$$
\mathbf{K}_{\mathbf{e}}=k_{x} \mathbf{i}+k_{y} \mathbf{j}+0 \mathbf{k},
$$

where

$$
k_{x}=\left|\mathbf{K}_{\mathbf{e}}\right| \cos \gamma, \quad k_{y}=\left|\mathbf{K}_{\mathbf{e}}\right| \sin \gamma
$$

and the diffraction condition (2.3) becomes

$(\cos \gamma, \sin \gamma, 0)$

$$
\cdot\left(\begin{array}{c}
h \cos \Theta-k \sin \Theta \\
(h \sin \Theta+k \cos \Theta) \cos \Phi-l \sin \Phi \\
(h \sin \Theta+k \cos \Theta) \sin \Phi+l \cos \Phi
\end{array}\right)=0 .
$$

We need to solve Eq. (2.10) for $\Theta$ to satisfy the diffraction condition. From Eq. (2.10) we have

$$
\begin{aligned}
h \cos \Theta-k \sin \Theta= & -\tan \gamma[(h \sin \Theta+k \cos \Theta) \\
& \times \cos \Phi-l \sin \Phi] .
\end{aligned}
$$

Substituting into Eq. (2.7)

$$
\mathbf{G}=\left(\begin{array}{c}
-\tan \gamma(Z \cos \Phi-l \sin \Phi) \\
Z \cos \Phi-l \sin \Phi \\
Z \sin \Phi+l \cos \Phi
\end{array}\right),
$$

where $Z=h \sin \Theta+k \cos \Theta$.

Since $|\mathbf{G}|^{2}=h^{2}+k^{2}+l^{2}=G^{2}$, we obtain

$\frac{1}{\cos ^{2} \gamma}(Z \cos \Phi-l \sin \Phi)^{2}+(Z \sin \Phi+l \cos \Phi)^{2}=G^{2}$.

Equation (2.13) can be solved as a quadratic equation to obtain the diffraction condition. First, we make a change of variables

$$
\begin{aligned}
& V(\Phi, \gamma)=[Z(\Phi, \gamma) \cos \Phi-l \sin \Phi] \frac{1}{\cos \gamma} \\
& U(\Phi, \gamma)=Z(\Phi, \gamma) \sin \Phi+l \cos \Phi .
\end{aligned}
$$

Then Eq. (2.13) becomes a set of equations

$$
\begin{aligned}
& U \cos \Phi-V \cos \gamma \sin \Phi=l \\
& V^{2}+U^{2}-G^{2}=0
\end{aligned}
$$

or

$(U \cos \Phi-l)^{2}+U^{2} \cos ^{2} \gamma \sin ^{2} \Phi-G^{2} \cos ^{2} \gamma \sin ^{2} \Phi=0$ $V^{2} \cos ^{2} \Phi+(V \cos \gamma \sin \Phi+l)^{2}-G^{2} \cos ^{2} \Phi=0$.

The solution of Eq. (2.16) is given by

$$
\begin{aligned}
& V_{ \pm}(\Phi, \gamma)=\frac{-l \cos \gamma \sin \Phi \pm \cos \Phi R}{S^{2}} \\
& U_{ \pm}(\Phi, \gamma)=\frac{l \cos \Phi \pm \cos \gamma \sin \Phi R}{S^{2}},
\end{aligned}
$$

where $S^{2}=1-\sin ^{2} \gamma \sin ^{2} \Phi$ and $R=\sqrt{-l^{2}+G^{2} S^{2}}$.

The reciprocal vector $\mathbf{G}$ satisfying the diffraction condition (2.3) in terms of $V_{ \pm}(\Phi, \gamma)$ and $U_{ \pm}(\Phi, \gamma)$ is given by

$$
\mathbf{G}(\Phi, \gamma)=\left(\begin{array}{c}
-V_{ \pm}(\Phi, \gamma) \sin \gamma \\
V_{ \pm}(\Phi, \gamma) \cos \gamma \\
U_{ \pm}(\Phi, \gamma)
\end{array}\right) .
$$

The diffraction pattern on the screen is then described by the following vector

$$
\mathbf{I}=\frac{d}{\left|\mathbf{K}_{\mathrm{e}}\right|}\left(\begin{array}{c}
G_{\|}(\Phi, \gamma) \\
G_{z}(\Phi, \gamma)
\end{array}\right)=\frac{d}{\left|\mathbf{K}_{\mathrm{e}}\right|} \frac{2 \pi}{a}\left(\begin{array}{c}
V_{ \pm}(\Phi, \gamma) \\
U_{ \pm}(\Phi, \gamma)
\end{array}\right),
$$

where $G_{\|}(\Phi, \gamma)$ is a component of $\mathbf{G}$ that is parallel to the $i j$ plane and $d$ is the perpendicular distance from the sample to the screen.

We point out here that for the cases when $\gamma=\frac{\pi}{2}$ and $\gamma$ $=0$ corresponding to the texture axis lying in the plane of specular reflection (the plane perpendicular to the film containing the incident beam) and the texture axis lying in the plane perpendicular to the plane of specular diffraction, respectively, Eq. (2.19) reproduces the solutions for the special cases considered by Andrieu et al. ${ }^{8}$

Equation (2.19) applies to the case of an ideal texture. To describe a nonideal asymmetric texture we let the preferred crystallographic direction rock around its average direction (texture axis). The quantities $\Delta \Phi_{\|}$and $\Delta \Phi_{\perp}$ define the components of the angular spread parallel and perpendicular to the $j k$ plane, respectively, with $\Delta \Phi_{\| 0}$ and $\Delta \Phi_{\perp 0}$ being the maximum values of these deviations such that

$$
\left(\frac{\Delta \Phi_{\|}}{\Delta \Phi_{\| 0}}\right)^{2}+\left(\frac{\Delta \Phi_{\perp}}{\Delta \Phi_{\perp 0}}\right)^{2} \leqslant 1
$$

It is straightforward to show that the component of the angular spread prependicular to the $j k$ plane, i.e., $\Delta \Phi_{\perp}$, is related to the quantity $\Delta \gamma=\gamma-\gamma_{0}$ by the following equation 


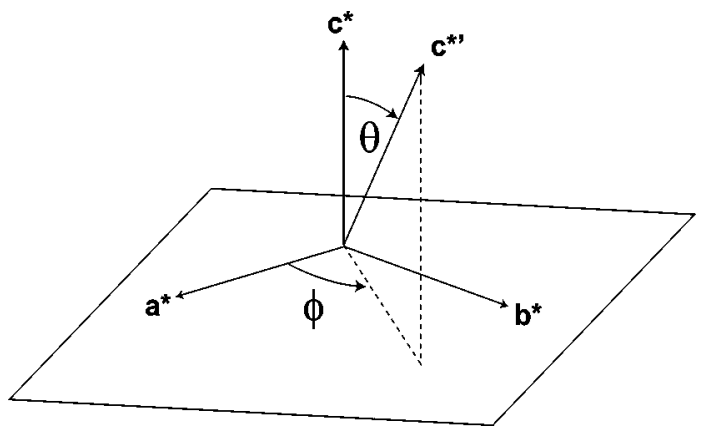

FIG. 4. Change of basis in reciprocal space.

$\tan \Delta \Phi_{\perp}=\tan \Delta \gamma \sin \Phi_{0}$,

where $\Phi_{0}$ and $\gamma_{0}$ are the values of $\Phi$ and $\gamma$, respectively, that define the orientation of the texture axis.

We will assume a Gaussian distribution in the angular dispersion of the texture. As stated earlier, we also assume that the total intensity at a particular spot in the diffraction pattern is a sum of intensities from different crystallites. Consequently, we can modify Eq. (2.19) to obtain the intensity distribution along the diffraction arc:

$$
\mathbf{I}(\Phi, \gamma)=A_{0} \frac{d}{\left|\mathbf{K}_{\mathrm{e}}\right|} \frac{2 \pi}{a}\left(\begin{array}{c}
V_{ \pm}(\Phi, \gamma) \\
U_{ \pm}(\Phi, \gamma)
\end{array}\right) D\left(\Delta \Phi_{\|}, \Delta \Phi_{\perp}\right),
$$

where $A_{0}$ is a normalizing constant and $D\left(\Delta \Phi_{\|}, \Delta \Phi_{\perp}\right)$ is a distribution function which in the case of a Gaussian distribution is

$$
\begin{aligned}
D\left(\Delta \Phi_{\|}, \Delta \Phi_{\perp}\right)= & \frac{2}{\pi} \frac{1}{\Delta \Phi_{\| 0} \Delta \Phi_{\perp 0}} \\
& \times \exp \left[-2\left(\frac{\Delta \Phi_{\|}^{2}}{\Delta \Phi_{\| 0}^{2}}+\frac{\Delta \Phi_{\perp}^{2}}{\Delta \Phi_{\perp 0}^{2}}\right)\right] .
\end{aligned}
$$

Equation (2.22) describes the RHEED pattern from a polycrystalline film with a texture. One should note that this equation also takes into account diffraction from a film without any texture present. In such a case one should set $\Delta \Phi_{0}$ equal to $\pi$ and $D\left(\Delta \Phi_{\|}, \Delta \Phi_{\perp}\right)$ equal to 1 .

\section{Arbitrary texture}

Having described diffraction for (001) texture, we can extend the formalism to a case with an arbitrary texture $\left(h_{0} k_{0} l_{0}\right)$. One can choose any set of three noncoplanar vectors as a basis to describe the reciprocal lattice. Here we choose an orthogonal basis set $\left\{\mathbf{a}^{*^{\prime}} \mathbf{b}^{*^{\prime}} \mathbf{c}^{*^{\prime}}\right\}$ such that the direction of the texture coincides with the direction of the $\mathbf{c}^{*^{\prime}}$ axis. Then the problem becomes exactly the same as in the case of (001) texture.

We use the following transformation to calculate reciprocal lattice indices in the new basis (Fig. 4)

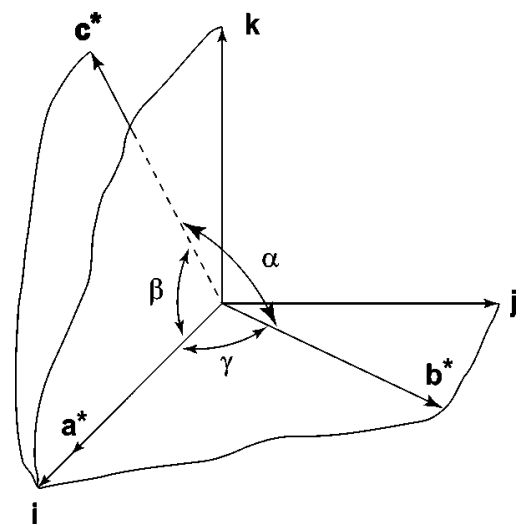

FIG. 5. Change of coordinates in triclinic system.

$$
\begin{aligned}
J_{h k l \rightarrow h^{\prime} k^{\prime} l^{\prime}}= & \left(\begin{array}{ccc}
\cos \omega & 0 & -\sin \omega \\
0 & 1 & 0 \\
\sin \omega & 0 & \cos \omega
\end{array}\right) \\
& \times\left(\begin{array}{ccc}
\cos \phi & \sin \phi & 0 \\
-\sin \phi & \cos \phi & 0 \\
0 & 0 & 1
\end{array}\right),
\end{aligned}
$$

or

$\left(\begin{array}{l}h^{\prime} \\ k^{\prime} \\ l^{\prime}\end{array}\right)=\left(\begin{array}{ccc}\cos \omega \cos \phi & \cos \omega \sin \phi & -\sin \omega \\ -\sin \phi & \cos \phi & 0 \\ \sin \omega \cos \phi & \sin \omega \sin \phi & \cos \omega\end{array}\right)\left(\begin{array}{l}h \\ k \\ l\end{array}\right)$,

where

$$
\cos \omega=\frac{l_{0}}{\sqrt{h_{0}^{2}+k_{0}^{2}+l_{0}^{2}}}, \quad \cos \phi=\frac{h_{0}}{\sqrt{h_{0}^{2}+k_{0}^{2}}} .
$$

To calculate the RHEED pattern for this case of arbitrary texture axis in an orthogonal crystal system, we use Eq. (2.22) replacing $h, k, l$ values with $h^{\prime}, k^{\prime}, l^{\prime}$ defined by Eq. (2.25).

\section{Case of nonorthogonal crystal axes}

So far, we have considered diffraction from films with an underlying orthogonal crystal lattice. Similar to the previous section where we discussed an arbitrary texture case, the choice of a particular basis is due purely to its convenience. We can always choose an orthogonal basis for any crystal symmetry and recalculate the new coordinates of reciprocal lattice points. The problem then becomes identical to the one described previously and all the results remain valid.

We will illustrate how this procedure should be applied using the triclinic system (see Fig. 5) as an example. $\mathbf{a}^{*}, \mathbf{b}^{*}$, and $\mathbf{c}^{*}$ are the basis vectors for the reciprocal crystal lattice, $\alpha, \beta$, and $\gamma$ are the interaxial angles, and $\mathbf{i}, \mathbf{j}$, and $\mathbf{k}$ are the vectors for the orthogonal basis. We will denote by $\omega_{\mathbf{i}, \mathbf{a}^{*}}, \omega_{\mathbf{i}, \mathbf{b}^{*}}, \ldots$, the angles $\overparen{\mathbf{i a} *}, \overparen{\mathbf{i} \mathbf{b}^{*}}, \ldots$. One can show that 


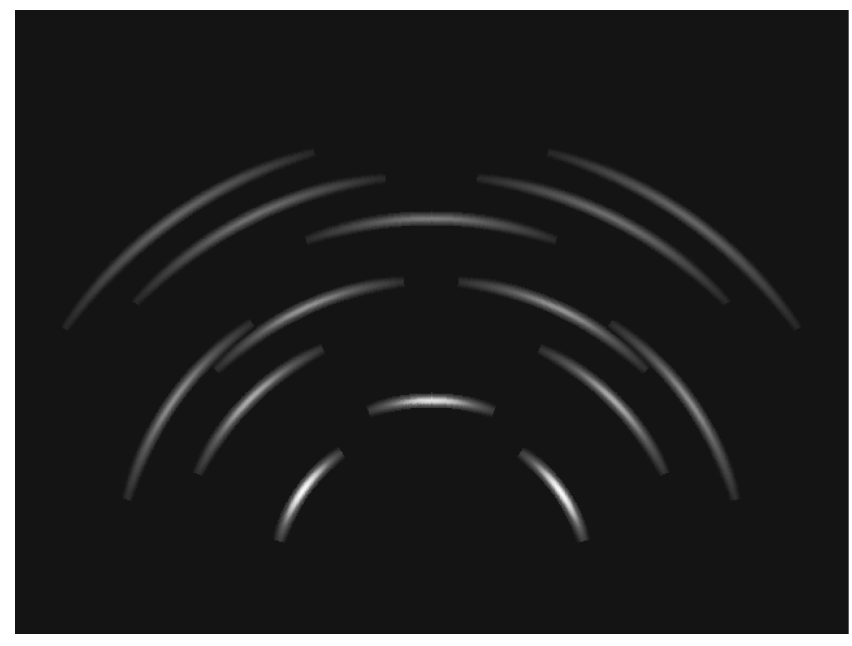

FIG. 6. Simulated RHEED pattern for rocksalt crystal lattice with (001) texture perpendicular to the plane of the film. Angular spread in texture is $15^{\circ}$

$$
\begin{aligned}
\cos \omega_{\mathbf{j}, \mathbf{c} *}= & \frac{\cos \gamma \cos \beta-\cos \alpha}{\sin \gamma} \\
\cos ^{2} \omega_{\mathbf{k}, \mathbf{c}^{*}} & \\
= & \frac{1+2 \cos \alpha \cos \beta \cos \gamma-\cos ^{2} \alpha-\cos ^{2} \beta-\cos ^{2} \gamma}{\sin ^{2} \gamma} .
\end{aligned}
$$

Then we have

$\mathbf{a}^{*}=a^{*} \mathbf{i}$

$\mathbf{b}^{*}=\left(b^{*} \cos \gamma\right) \cdot \mathbf{i}+\left(b^{*} \sin \gamma\right) \mathbf{j}$

$\mathbf{c}^{*}=\left(c^{*} \cos \beta\right) \cdot \mathbf{i}+\left(c^{*} \cos \omega_{\mathbf{j}, \mathbf{c} *}\right) \cdot \mathbf{j}+\left(c^{*} \cos \omega_{\mathbf{k}, \mathbf{c}} *\right) \cdot \mathbf{k}$,

where $a^{*}=\left|\mathbf{a}^{*}\right|, b^{*}=\left|\mathbf{b}^{*}\right|$, and $c^{*}=\left|\mathbf{c}^{*}\right|$.

The required expression for $\mathbf{G}$ in the new coordination system is obtained by substituting expressions for $\mathbf{a}^{*}, \mathbf{b}^{*}$, and $\mathbf{c}^{*}$ from Eq. (2.28) into Eq. (2.2):

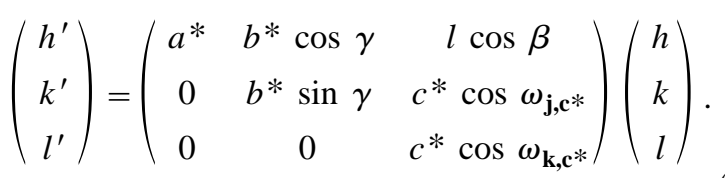

Having redefined the coordinate system to the orthogonal one, we shall apply the procedure described above to align the texture along the (001) axis and use Eq. (2.22) to calculate the diffraction pattern.

\section{ANALYSIS AND DISCUSSION}

\section{A. Texture axis not in the plane of the film}

Let us consider a case where the texture axis is parallel to the plane of specular diffraction (see Sec. II A), i.e., $\gamma_{0}$ $=\pi / 2$. From Eq. (2.17)

$$
\begin{aligned}
& U_{+}\left(\Phi, \frac{\pi}{2} \pm \Delta \gamma\right)=U_{-}\left(\Phi, \frac{\pi}{2} \mp \Delta \gamma\right) \\
& V_{+}\left(\Phi, \frac{\pi}{2} \pm \Delta \gamma\right)=-V_{-}\left(\Phi, \frac{\pi}{2} \mp \Delta \gamma\right) .
\end{aligned}
$$

It follows that in this case the RHEED pattern defined by Eq. (2.19) is symmetric with respect to the $z$ axis.

Next, consider the case where the texture axis is perpendicular to the film surface. We set $\gamma=0$. Then from Eq. (2.17) we have

$$
\begin{aligned}
& U_{+}( \pm \Delta \Phi, 0)=U_{-}(\mp \Delta \Phi, 0) \\
& V_{+}( \pm \Delta \Phi, 0)=-V_{-}(\mp \Delta \Phi, 0) .
\end{aligned}
$$

It follows that in the case of the texture axis perpendicular to the surface of the film, the RHEED pattern will always be symmetric with respect to the $z$ axis and it is invariant with respect to the sample rotation around the $z$-axis.

Let us consider now a case where $\mathbf{G}=(0,0, l)$, i.e., $\mathbf{G}$ along the texture direction. Then $R=l \sqrt{S^{2}-1}$. Thus, for the solution to exist $S^{2}$ must be greater or equal to 1 . By definition, $S^{2} \leqslant 1$. Combining the two inequalities we get

$$
S^{2}=1, \quad R=0
$$

and

$$
\sin \gamma=0 \text { or } \sin \Phi=0,
$$

and Eq. (2.22) becomes

$$
\mathbf{I}=A_{0} \frac{d}{\left|\mathbf{K}_{\mathrm{e}}\right|} \frac{2 \pi}{a} l\left(\begin{array}{c}
-\cos \gamma \sin \Phi \\
\cos \Phi
\end{array}\right) D\left(\Delta \Phi_{\|}, \Delta \Phi_{\perp}\right) .
$$

When $\sin \Phi=0$, corresponding to an ideal texture perpendicular to the film surface, Eq. (3.4) gives just one point of diffraction and, as will be clear below, this is a subcase of $\sin \gamma=0$. When $\sin \gamma=0$, i.e., when the texture lies in the plane perpendicular to the plane of specular diffraction, Eq. (2.22) becomes

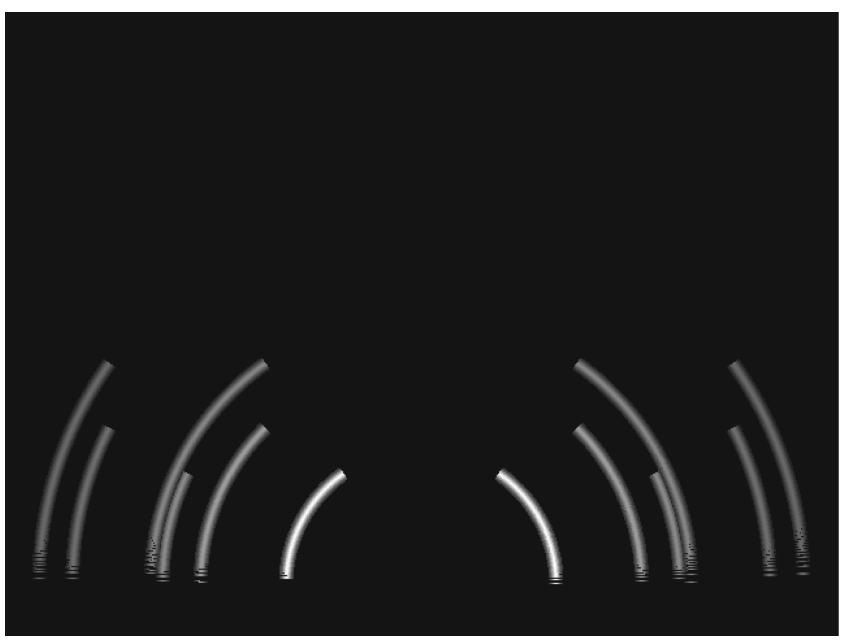

FIG. 7. Simulated RHEED image of double texture for rocksalt crystal lattice. 


$$
\mathbf{I}=A_{0} \frac{d}{\left|\mathbf{K}_{\mathbf{e}}\right|} \frac{2 \pi}{a} l\left(\begin{array}{c} 
\pm \sin \Phi \\
\cos \Phi
\end{array}\right) D\left(\Delta \Phi_{\|}, \Delta \Phi_{\perp}\right) .
$$

This is the equation of an arc with its intensity distribution symmetric with respect to the $z$ axis and with a maximum of intensity on the $z$ axis. This is consistent with the discussion above (see Sec. II A) where the presence of a diffraction arc with such an intensity distribution indicates the presence of texture in the direction of the particular $\mathbf{G}$ responsible for that specific diffraction arc (see Fig. 6).

\section{B. Texture parallel to the surface of the film}

The final example is when the texture axis lies in the plane of the sample surface. Equation (2.22) then becomes

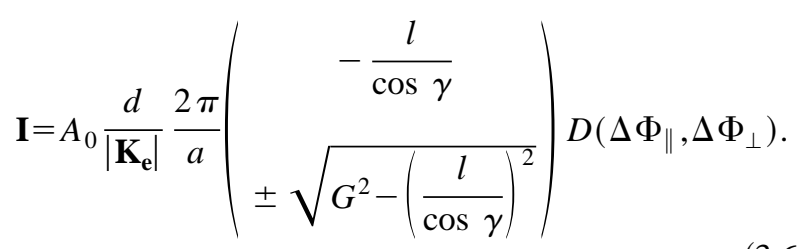

For the case of "double texture," where the texture axis is free to rotate within the plane of the film, Eq. (3.6) gives the corresponding diffraction pattern if $\gamma$ is varied from $-\pi$ to $\pi$ (see Fig. 7).

\section{CONCLUSIONS}

We have presented a kinematic theory of RHEED for a polycrystalline film with an arbitrary degree of crystallite ordering. The analytical expressions developed allow simulation of RHEED patterns for polycrystalline films with arbitrary textures and arbitrary underlying crystal lattices. It is shown how the developed formalism can be applied to texture identification and angular dispersion characterization. The approach presented here should be useful for in situ texture characterization of thin films facilitating the choice of optimum deposition conditions.

${ }^{1}$ J. Harris, B. Joyce, and P. Dobson, Surf. Sci. 103, L90 (1981).

${ }^{2}$ B. Joyce, J. Neave, J. Zhang, P. Dobson, P. Dawson, K. Moore, and C. Foxon, in Thin Film Growth Techniques for Low-Dimensional Structures (Plenum, New York, 1987), pp. 19-35.

${ }^{3}$ J. E. Mahan, K. M. Geib, G. Robinson, and R. G. Long, J. Vac. Sci. Technol. A 8, 3692 (1990).

${ }^{4}$ A. Arrott, in Ultrathin Magnetic Structures, edited by J. Bland and B. Heinrich (Springer, Berlin, 1994), Vol. 1, pp. 177-303.

${ }^{5}$ Y. Wang, Q. Tang, Z. Wang, H. Fan, S. Fan, and F. Li, J. Magn. Magn. Mater. 51, 315 (1985).

${ }^{6}$ C. Wang, K. Do, M. Beasley, T. Geballe, and R. Hammond, Appl. Phys. Lett. 71, 2955 (1997).

${ }^{7}$ D. Litvinov and R. Clarke, Appl. Phys. Lett. 71, 1969 (1997).

${ }^{8}$ S. Andrieu and P. Frechard, Surf. Sci. 360, 289 (1996). 